International Journal of Pure and Applied Mathematics

Volume 107 No. $3 \quad 2016,551-563$

ISSN: 1311-8080 (printed version); ISSN: 1314-3395 (on-line version)

url: http://www.ijpam.eu

doi: 10.12732/ijpam.v107i3.4

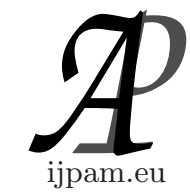

\title{
FINITE AND INFINITE MULTI-SERIES TYPE SOLUTIONS OF GENERALIZED MIXED DIFFERENCE EQUATION
}

\author{
M. Maria Susai Manuel ${ }^{1}$, G. Dominic Babu ${ }^{2}$, G. Britto Antony Xavier ${ }^{3}$ \\ ${ }^{1}$ Department of Mathematics \\ R.M.D. Engineering College \\ Kavaraipettai, 601 206, Tamil Nadu, S. INDIA \\ ${ }^{2}$ Department of Mathematics \\ Annai Velankanni College \\ Tholaiyavattam, Kanyakumari District, Tamil Nadu, S. INDIA \\ ${ }^{3}$ Department of Mathematics \\ Sacred Heart College \\ Tirupattur, Vellore District, INDIA
}

\begin{abstract}
In this paper, we obtain the solutions of a generalized $\alpha_{i}$-mixed difference equation in closed, finite and infinite multi-series forms. By equating closed form with multi-series type solutions of the $\alpha_{i}$-mixed difference equation, we obtain the values of certain types of infinite and finite multi-series. Suitable examples are provided to illustrate the main results.
\end{abstract}

AMS Subject Classification: 39A70, 47B39, 39A10

Key Words: generalized difference equation, mixed difference equation, solutions in multiseries, closed form solution

\section{Introduction}

In 1984, Jerzy Popenda [5] introduced a particular type of difference operator $\Delta_{\alpha}$ defined on $u(k)$ as $\Delta_{\alpha} u(k)=u(k+1)-\alpha u(k)$. In 1989, Miller and Rose [9] introduced the discrete analogue of the Riemann-Liouville fractional derivative

Received: January 5, 2016

Published: April 19, 2016

${ }^{\S}$ Correspondence author
(C) 2016 Academic Publications, Ltd.

url: www.acadpubl.eu 
and proved some properties of the fractional difference operator. The general fractional h-difference Riemann-Liouville operator and its inverse $\Delta_{h}^{-\nu} f(t)$ were mentioned in [1, 2]. In 2011, M.Maria Susai Manuel, et.al, [8, 11] extended the definition of $\Delta_{\alpha}$ to $\Delta_{\alpha(\ell)}$ defined on $u(k)$ as $\Delta_{\alpha(\ell)} v(k)=v(k+\ell)-\alpha v(k)$, where $\alpha \neq 0, \ell>0$ are fixed and $k \in[0, \infty)$ is a variable. The results derived in [11] coincide with the results in [7] when $\alpha=1$.

An equation involving both $\Delta$ and $\Delta_{\alpha}$ is called a mixed difference equation. Oscillatory behaviour of solutions for a few mixed difference equations were discussed in $[3,4,6,12]$. An equation involving $\Delta_{\ell}$ and $\Delta_{\alpha(\ell)}$ is called as the generalized mixed difference equation.

The higher order generalized $\alpha_{i}$-difference equation

$$
\Delta_{\alpha_{1}\left(\ell_{1}\right)}\left(\Delta_{\alpha_{2}\left(\ell_{2}\right)}\left(\cdots \Delta_{\alpha_{n}\left(\ell_{n}\right)}(v(k)) \cdots\right)\right)=u(k), \quad k \in[0, \infty), \ell_{i}>0 \alpha_{i} \neq 0
$$

becomes a generalized mixed difference equation if $\alpha_{i}=1$ for some $i$ and $n \geq 2$. The equation (1) has three types of solutions which are closed, finite and infinite multi-series forms.

\section{Preliminaries}

In this section, we present some basic definitions, results and examples on $\alpha_{i^{-}}$ mixed difference equation. Throughout this paper, we assume that $k \in[0, \infty), \ell_{i}>0, \alpha_{i} \neq 0$ and we denote $\Delta_{\alpha_{i}\left(\ell_{i}\right)} v(k)=v\left(k+\ell_{i}\right)-\alpha_{i} v(k)$, $\hat{\ell}_{i}(k)=k-\left[\frac{k}{\ell_{i}}\right] \ell_{i}$.

Definition 2.1. [11] If there exists a function $v(k)$ such that $\Delta_{\alpha_{i}\left(\ell_{i}\right)} v(k)=$ $u(k)$, then $v(k)=\Delta_{\alpha_{i}\left(\ell_{i}\right)}^{-1} u(k)$ is a solution of the first order generalized $\alpha_{i^{-}}$ difference equation $v\left(k+\ell_{i}\right)-\alpha_{i} v(k)=u(k)$.

Example 2.2. The first order difference equation $v\left(k+\ell_{i}\right)-\alpha_{i} v(k)=a^{s k}$ has a closed form solution $\Delta_{\alpha_{i}\left(\ell_{i}\right)}^{-1} a^{s k}=\frac{a^{s k}}{a^{s \ell_{i}}-\alpha_{i}}$ when $a^{s \ell_{i}}-\alpha_{i} \neq 0$. Here $u(k)=a^{s k}$ and $v(k)=\frac{a^{s k}}{a^{s \ell_{i}}-\alpha_{i}}$. The solution in infinite series form of the first order difference equation is given in Example 2.6. 
Lemma 2.3. If $a^{s \ell_{i}}-\alpha_{i} \neq 0$ for $i=1,2, \ldots, n$, then

$$
\prod_{i=1}^{n} \Delta_{\alpha_{i}\left(\ell_{i}\right)}^{-1} a^{s k}=\frac{a^{s k}}{\prod_{i=1}^{n}\left(a^{s \ell_{i}}-\alpha_{i}\right)}
$$

is a closed form solution of the equation (1) when $u(k)=a^{s k}$ and hence $\Delta_{\alpha(\ell)}^{n} v(k)=a^{s k}$ has a closed form solution

$$
\Delta_{\alpha(\ell)}^{-n} a^{s k}=\frac{a^{s k}}{\left(a^{s \ell}-\alpha\right)^{n}}
$$

Proof. Since $\Delta_{\alpha_{i}\left(\ell_{i}\right)}^{-1} v(k)$ is linear, $a^{s \ell_{i}}-\alpha_{i}$ is constant and $\Delta_{\alpha_{i}\left(\ell_{i}\right)}^{-1} a^{s k}=$ $\frac{a^{s k}}{a^{s \ell_{i}}-\alpha_{i}}$, the relation (2) follows by operating $\Delta_{\alpha_{1}\left(\ell_{1}\right)}^{-1}, \Delta_{\alpha_{2}\left(\ell_{2}\right)}^{-1}, \ldots, \Delta_{\alpha_{n}\left(\ell_{n}\right)}^{-1}$ on $a^{s k}$.

Now (3) is obtained by taking $\alpha_{i}=\alpha$ and $\ell_{i}=\ell$ for $i=1,2, \ldots, n$ in $(2)$.

Lemma 2.4. [11] The first order generalized $\alpha$-difference equation

$$
v(k+\ell)-\alpha v(k)=u(k), k \in[0, \infty), \ell \in(0, \infty)
$$

has a solution in the finite summation form as

$$
v(k)=\Delta_{\alpha(\ell)}^{-1} u(k)-\alpha^{\left[\frac{k}{\ell}\right]} \Delta_{\alpha(\ell)}^{-1} u(\hat{\ell}(k))=\sum_{r=1}^{\left[\frac{k}{\ell}\right]} \alpha^{r-1} u(k-r \ell),
$$

where $\hat{\ell}(k)=k-\left[\frac{k}{\ell}\right] \ell,\left[\frac{k}{\ell}\right]$ is the integer part of $\frac{k}{\ell}$.

Lemma 2.5. If $\lim _{r_{i} \rightarrow \infty} \frac{1}{\alpha_{i}^{r_{i}}} \Delta_{\alpha_{i}\left(\ell_{i}\right)}^{-1} u\left(k+r_{i} \ell_{i}\right)=0$, then the $\alpha_{i}$-difference equation

$$
\Delta_{\alpha_{i}\left(\ell_{i}\right)} v(k)=u(k), \quad k \in[0, \infty), \ell_{i}>0,
$$

has a solution in the infinite series form as

$$
\Delta_{\alpha_{i}\left(\ell_{i}\right)}^{-1} u(k)=\frac{-1}{\alpha_{i}} \sum_{r_{i}=0}^{\infty} \alpha_{i}^{-r_{i}} u\left(k+r_{i} \ell_{i}\right)
$$


Proof. Taking $\alpha=\alpha_{i}$ and $\ell=\ell_{i}$ in (4), we get

$$
v\left(k+\ell_{i}\right)-\alpha_{i} v(k)=u(k)
$$

which yields

$$
v(k)=-\frac{1}{\alpha_{i}} u(k)+\frac{1}{\alpha_{i}} v\left(k+\ell_{i}\right) .
$$

Replacing $k$ by $k+l_{i}$ in (8), we obtain

$$
v\left(k+\ell_{i}\right)=-\frac{1}{\alpha_{i}} u\left(k+\ell_{i}\right)+\frac{1}{\alpha_{i}} v\left(k+2 \ell_{i}\right),
$$

which yields

$$
v(k)=-\frac{1}{\alpha_{i}} u(k)-\frac{1}{\alpha_{i}^{2}} u\left(k+\ell_{i}\right)+\frac{1}{\alpha_{i}^{2}} v\left(k+2 \ell_{i}\right) .
$$

From $(9), \Delta_{\alpha_{i}\left(\ell_{i}\right)}^{-1} u(k)=v(k), \lim _{\alpha_{i} \rightarrow \infty} \frac{1}{\alpha_{i}^{r_{i}}} v\left(k+r_{i} \ell_{i}\right)=0$ and by replacing $k$ by $k+2 \ell_{i}, k+3 \ell_{i}, \ldots$ in $(8)$, we arrive $(7)$.

The following example illustrates the existence of the solution in the infinite series form.

Example 2.6. If $\ell_{i}>0, \alpha_{i} \geq 1, a>1$ and $s<0$, then we have

$$
\frac{\alpha_{i} a^{s k}}{\alpha_{i}-a^{s \ell_{i}}}=\sum_{r_{i}=0}^{\infty} \alpha_{i}^{-r_{i}} a^{s\left(k+r_{i} \ell_{i}\right)} \text { fork } \in(-\infty, \infty) \text {. }
$$

Proof. The proof follows by equating the infinite series form solution given in Lemma 2.5 and the closed form solution in Example 2.2.

\section{Main Results}

In this section, we obtain the values of certain types of finite and infinite alpha multi-series by equating the closed form with finite and infinite multi-series solutions of the generalized $\alpha_{i}$-difference equation (1). Suitable examples are presented for second order mixed difference equation.

Definition 3.1. The solution of the form

$$
v(k)=\sum_{r_{1}=0}^{\infty} \sum_{r_{2}=0}^{\infty} \cdots \sum_{r_{n}=0}^{\infty} \frac{u\left(k+\sum_{i=1}^{n} r_{i} \ell_{i}\right)}{\alpha_{1}^{r_{1}} \alpha_{2}^{r_{2}} \cdots \alpha_{n}^{r_{n}}}
$$


is called an infinite multi-series solution of the equation (1) and the summation of the form

$$
v(k)=\sum_{r_{1}=0}^{\left[\frac{k}{\ell_{1}}\right]} \sum_{r_{2}=0}^{\left[\frac{k-r_{1} \ell_{1}}{\ell_{2}}\right]} \cdots \sum_{r_{n}=0}^{\left[\frac{k-r_{1} \ell_{1}-\ldots-r_{n-1} \ell_{n-1}}{\ell_{n}}\right.} \alpha_{1}^{r_{1}} \alpha_{2}^{r_{2}} \cdots \alpha_{n}^{r_{n}} u\left(k-r_{1} \ell_{1}-\ldots-r_{n} \ell_{n}\right)
$$

is called a finite multi-series solution of the equation (1).

If there exists a function $v(k)$, other than the finite and infinite multiseries solutions, such that $\Delta_{\alpha_{1}\left(\ell_{1}\right)} \Delta_{\alpha_{2}\left(\ell_{2}\right)} \cdots \Delta_{\alpha_{n}\left(\ell_{n}\right)} v(k)=u(k)$, then $v(k)=$ $\Delta_{\alpha_{1}\left(\ell_{1}\right)}^{-1} \Delta_{\alpha_{2}\left(\ell_{2}\right)}^{-1} \cdots \Delta_{\alpha_{n}\left(\ell_{n}\right)}^{-1} u(k)$ is called a closed form solution of the $\alpha_{i}$-difference equation (1).

Example 3.2. The first order $\alpha$-difference equation $\Delta_{\alpha(\ell)} v(k)=\frac{1}{k}, k>0$ has neither closed form nor infinite series solution in any closed interval but it has a finite series solution $v(k)=\sum_{r=0}^{\left[\frac{k}{\ell}\right]} \alpha^{r-1} \frac{1}{k-r \ell}$ for $k \in(\ell, \infty), \ell>0$.

Theorem 3.3. (Infinite alpha multi-series) If for $t=1,2, \ldots, n$,

$$
\lim _{r_{1} \rightarrow \infty}\left\{\frac{1}{\alpha_{1}^{r_{1}}} \prod_{i=1}^{t} \Delta_{\alpha_{i+1}\left(\ell_{i+1}\right)}^{-1} u\left(k+r_{1} \ell_{1}\right)\right\}=0
$$

then the summation of the form

$$
\sum_{r_{1}=0}^{\infty} \sum_{r_{2}=0}^{\infty} \ldots \sum_{r_{n}=0}^{\infty} \frac{u\left(k+\sum_{i=1}^{n} r_{i} \ell_{i}\right)}{\alpha_{1}^{r_{1}} \alpha_{2}^{r_{2}} \ldots \alpha_{n}^{r_{n}}}=\prod_{i=1}^{n}\left(-\alpha_{i}\right) \Delta_{\alpha_{i}\left(\ell_{i}\right)}^{-1} u(k)
$$

is an infinite multi-series solution of the equation (1).

Proof. From the Lemma 2.5, we have

$$
u(k)+\frac{1}{\alpha_{2}} u\left(k+\ell_{2}\right)+\frac{1}{\alpha_{2}^{2}} u\left(k+2 \ell_{2}\right)+\ldots \infty=-\alpha_{2} \Delta_{\alpha_{2}\left(\ell_{2}\right)}^{-1} u(k) .
$$

Dividing (12) by $\alpha_{1}^{r_{1}}$ and replacing $k$ by $k+r_{1} \ell_{1}$, we get

$$
\frac{1}{\alpha_{1}^{r_{1}}}\left\{u\left(k+r_{1} \ell_{1}\right)+\frac{1}{\alpha_{2}} u\left(k+r_{1} \ell_{1}+l_{2}\right)+\frac{1}{\alpha_{2}^{2}} u\left(k+r_{1} \ell_{1}+2 \ell_{2}\right)+\ldots+\infty\right\}
$$




$$
=\frac{1}{\alpha_{1}^{r_{1}}}\left(-\alpha_{2}\right) \Delta_{\alpha_{2}\left(\ell_{2}\right)}^{-1} u\left(k+r_{1} \ell_{1}\right) .
$$

Adding (13) for each $r_{1}=0,1,2, \ldots$ and then applying Lemma 2.5, we get

$$
\sum_{r_{1}=0}^{\infty} \sum_{r_{2}=0}^{\infty} \frac{u\left(k+r_{1} \ell_{1}+r_{2} \ell_{2}\right)}{\alpha_{1}^{r_{1}} \alpha_{2}^{r_{2}}}=\left(-\alpha_{1}\right)\left(-\alpha_{2}\right) \Delta_{\alpha_{1}\left(\ell_{1}\right)}^{-1} \Delta_{\alpha_{2}\left(\ell_{2}\right)}^{-1} u(k) .
$$

Replacing $r_{1}$ by $r_{2}, r_{2}$ by $r_{3}, \ell_{1}$ by $\ell_{2}$ and $\ell_{2}$ by $\ell_{3}$, in (14), we obtain

$$
\sum_{r_{2}=0}^{\infty} \sum_{r_{3}=0}^{\infty} \frac{u\left(k+r_{2} \ell_{2}+r_{3} \ell_{3}\right)}{\alpha_{2}^{r_{2}} \alpha_{3}^{r_{3}}}=\left(-\alpha_{2}\right)\left(-\alpha_{3}\right) \Delta_{\alpha_{2}\left(\ell_{2}\right)}^{-1} \Delta_{\alpha_{3}\left(\ell_{3}\right)}^{-1} u(k) .
$$

Dividing (15) by $\alpha_{1}^{r_{1}}$ and replacing $k$ by $k+r_{1} \ell_{1}$, we obtain

$$
\frac{1}{\alpha_{1}^{r_{1}}} \sum_{r_{2}=0}^{\infty} \sum_{r_{3}=0}^{\infty} \frac{u\left(k+r_{1} l_{1}+r_{2} l_{2}+r_{3} l_{3}\right)}{\alpha_{2}^{r_{2}} \alpha_{3}^{r_{3}}}=\frac{\alpha_{2} \alpha_{3}}{\alpha_{1}^{r_{1}}} \Delta_{\alpha_{2}\left(l_{2}\right)}^{-1} \Delta_{\alpha_{3}\left(l_{3}\right)}^{-1} u\left(k+r_{1} l_{1}\right) .
$$

Adding (16) for each $r_{1}=0,1,2, \ldots$ and then applying Lemma 2.5, we arrive

$$
\sum_{r_{1}=0}^{\infty} \sum_{r_{2}=0}^{\infty} \sum_{r_{3}=0}^{\infty} \frac{u\left(k+r_{1} l_{1}+r_{2} l_{2}+r_{3} l_{3}\right)}{\alpha_{1}^{r_{1}} \alpha_{2}^{r_{2}} \alpha_{3}^{r_{3}}}=-\alpha_{1} \alpha_{2} \alpha_{3} \Delta_{\alpha_{1}\left(l_{1}\right)}^{-1} \Delta_{\alpha_{2}\left(l_{2}\right)}^{-1} \Delta_{\alpha_{3}\left(l_{3}\right)}^{-1} u(k) .
$$

Hence (11) follows by continuing this process.

Corollary 3.4. If $\alpha_{i}>1$ and $s<0$, then the equation (1) for $u(k)=2^{s k}$ has both infinite alpha multi-series and closed form solutions

$$
\begin{aligned}
\prod_{i=1}^{n}\left(-\alpha_{i}\right) \Delta_{\alpha_{i}\left(\ell_{i}\right)}^{-1} 2^{s k}=\sum_{r_{1}=0}^{\infty} \sum_{r_{2}=0}^{\infty} \cdots \sum_{r_{n}=0}^{\infty} \frac{(2)^{s\left(k+\sum_{i=1}^{n} r_{i} \ell_{i}\right)}}{\alpha_{1}^{r_{1}} \alpha_{2}^{r_{2}} \cdots \alpha_{n}^{r_{n}}} & \\
& =\prod_{i=1}^{n}\left(\frac{\alpha_{i}}{\alpha_{i}-2^{s \ell_{i}}}\right) 2^{s k}
\end{aligned}
$$

Proof. The proof follows by taking $u(k)=2^{\text {sk }}$ and $n=2$ in (11) and (2).

Example 3.5. Taking $n=2, k=10, \ell_{1}=4, \ell_{2}=5, \alpha_{1}=2, \alpha_{2}=3$ and $s=-1$ in (17), we get

$$
\sum_{r_{1}=0}^{\infty} \sum_{r_{2}=0}^{\infty} \frac{(2)^{-\left(10+4 r_{1}+5 r_{2}\right)}}{(2)^{r_{1}}(3)^{r_{2}}}=\frac{(2)(3)(2)^{-10}}{\left(2^{-4}-2\right)\left(2^{-5}-3\right)}=1.018675722 \times 10^{-3} .
$$


Remark 3.6. For some $i$, by putting $\alpha_{i}=1$ in (11), we get solution of mixed $\alpha_{i}$-difference equation.

Corollary 3.7. (Infinite multi-series) If $\lim _{r_{i} \rightarrow \infty} u\left(k+r_{i} \ell_{i}\right)=0$, then we have

$$
\sum_{r_{1}=0}^{\infty} \sum_{r_{2}=0}^{\infty} \ldots \sum_{r_{n}=0}^{\infty} u\left(k+\sum_{i=1}^{n} r_{i} \ell_{i}\right)=(-1)^{n} \prod_{i=1}^{n} \Delta_{\ell_{i}}^{-1} u(k),
$$

which is an infinite multi-series solution of the equation (1) for each $\alpha_{i}=1$.

Proof. The proof follows by taking each $\alpha_{i}=1$ in (11).

Example 3.8. For $s>0, a>1, \ell_{i}>0$ and $k \in(-\infty, \infty)$, we have

$$
\prod_{i=1}^{n} \frac{\Delta_{\ell_{i}}^{-1} a^{-s k}}{(-1)^{n}}=\sum_{r_{1}=0}^{\infty} \sum_{r_{2}=0}^{\infty} \cdots \sum_{r_{n}=0}^{\infty} a^{-s\left(k+\sum_{i=1}^{n} r_{i} \ell_{i}\right)}=\frac{a^{-s k}}{\prod_{i=1}^{n}\left(1-a^{-s \ell_{i}}\right)} .
$$

Proof. The Proof follows by replacing $u(k)$ by $a^{-s k}$ in (18) and using (2).

Corollary 3.9. (Infinite series) If $\lim _{r \rightarrow \infty} u(k+r \ell)=0$ and $(x)^{(n)}$ denotes the polynomial factorial $x(x-1) \ldots(x-(n-1))$, then we have

$$
\sum_{r=0}^{\infty} \frac{(r+(n-1))^{(n-1)}}{(n-1)} u(k+r \ell)=(-1)^{n} \Delta_{\ell}^{-n} u(k),
$$

which is an infinite series solution of the difference equation $\Delta_{\ell}^{n} v(k)=u(k)$.

Proof. Taking $\ell_{i}=\ell$ in (18), we get

$$
\sum_{r_{1}=0}^{\infty} \sum_{r_{2}=0}^{\infty} \ldots \sum_{r_{n}=0}^{\infty} u\left(k+\sum_{i=1}^{n} r_{i} \ell\right)=(-1)^{n} \Delta_{\ell}^{-n} u(k)
$$

By rearranging the terms, we obtain

$$
\sum_{r_{1}=0}^{\infty} \sum_{r_{2}=0}^{\infty} \ldots \sum_{r_{n}=0}^{\infty} u\left(k+\sum_{i=1}^{n} r_{i} \ell\right)=\sum_{r=0}^{\infty} \frac{(r+(n-1))^{(n-1)}}{(n-1)} u(k+r \ell) .
$$

By Substituting (22) in (21), we get (20).

The following example gives the value of an infinite series. 
Example 3.10. If $a>1, \ell>0$ and $s<0$, then for $k \in(-\infty, \infty)$ we have

$$
\sum_{r=0}^{\infty} \frac{(r+(n-1))^{(n-1)}}{(n-1)} a^{s(k+r \ell)}=\frac{a^{s k}}{\left(1-a^{s \ell}\right)^{n}}
$$

In particular taking $k=0$ in (23), we get

$$
\sum_{r=0}^{\infty} \frac{(r+(n-1))^{(n-1)}}{(n-1)} a^{s(r \ell)}=\frac{1}{\left(1-a^{s \ell}\right)^{n}}
$$

Proof. By taking $u(k)=a^{s k}$ in (20) and on putting $\alpha=1$ in (3), we get (23).

Theorem 3.11. If $\lim _{r_{i} \rightarrow \infty} \frac{1}{\alpha_{i}^{r_{i}}} \Delta_{\alpha_{i}\left(\ell_{i}\right)}^{-1} u\left(k+r_{i} \ell_{i}\right)=0$ for $i=1,2$, then we have

$$
\sum_{r_{1}=0}^{\infty} \sum_{r_{2}=0}^{r_{1}} \frac{1}{\alpha_{1}^{r_{1}-r_{2}} \alpha_{2}^{r_{2}}} u\left(k+\left(r_{1}-r_{2}\right) \ell_{1}+r_{2} \ell_{2}\right)=\alpha_{1} \alpha_{2} \Delta_{\alpha_{1}\left(\ell_{1}\right)}^{-1} \Delta_{\alpha_{2}\left(\ell_{2}\right)}^{-1} u(k) .
$$

In particular, the mixed difference equation $\Delta_{\ell_{2}} \Delta_{\alpha_{1}\left(\ell_{1}\right)} v(k)=u(k)$ has a solution in infinite series form as

$$
\sum_{r_{1}=0}^{\infty} \sum_{r_{2}=0}^{r_{1}} \frac{u\left(k+\left(r_{1}-r_{2}\right) \ell_{1}+r_{2} \ell_{2}\right)}{\alpha_{1}^{r_{1}-r_{2}+1}}=\Delta_{\alpha_{1}\left(\ell_{1}\right)}^{-1} \Delta_{\left(\ell_{2}\right)}^{-1} u(k) .
$$

Proof. Adding (13) for each $r_{1}=0,1,2, \ldots$ in the proof of Theorem 3.3 and grouping the terms, we arrive

$$
\begin{array}{r}
u(k)+\left(\frac{u\left(k+\ell_{1}\right)}{\alpha_{1}}+\frac{u\left(k+\ell_{2}\right)}{\alpha_{2}}\right)+\left(\frac{u\left(k+2 \ell_{1}\right)}{\alpha_{1}^{2}}+\frac{u\left(k+\ell_{1}+\ell_{2}\right)}{\alpha_{1} \alpha_{2}}+\frac{u\left(k+2 \ell_{2}\right)}{\alpha_{2}^{2}}\right) \\
+\left(\frac{u\left(k+3 \ell_{1}\right)}{\alpha_{1}^{3}}+\frac{u\left(k+2 \ell_{1}+\ell_{2}\right)}{\alpha_{1}^{2} \alpha_{2}}+\frac{u\left(k+\ell_{1}+2 \ell_{2}\right)}{\alpha_{1} \alpha_{2}^{2}}+\frac{u\left(k+3 \ell_{2}\right)}{\alpha_{2}^{3}}\right)+\ldots+\infty \\
=\left(\alpha_{1}\right)\left(\alpha_{2}\right) \Delta_{\alpha_{1}\left(\ell_{1}\right)}^{-1} \Delta_{\alpha_{2}\left(\ell_{2}\right)}^{-1} u(k),
\end{array}
$$

which gives (25). Now (26) is obtained by putting $\alpha_{2}=1$ in (25).

Theorem 3.11 yields the following infinite series form solution for the mixed difference equation $\Delta_{\ell_{2}} \Delta_{\alpha_{1}\left(\ell_{1}\right)} v(k)=u(k), \ell_{1}, \ell_{2}>0$. Next, we present finite multi-series solution of equation (1). 
Theorem 3.12. (Finite multi-series formula) If $k \in\left(\sum \ell_{i}, \infty\right)$, then the equation (1) has a solution in finite multi-series form as

$$
\begin{array}{r}
\sum_{i=1}^{n}\left\{\sum _ { ( r \ell ) _ { 1 \rightarrow i } } ^ { [ k ] } \prod _ { j = 1 } ^ { i } \alpha _ { j } ^ { r _ { j } } \alpha _ { i + 1 } ^ { [ \frac { k - \sum _ { j = 1 } ^ { i } r _ { j } \ell _ { j } } { \ell _ { i + 1 } } ] + 1 } \prod _ { j = i + 1 } ^ { n } \Delta _ { \alpha _ { j } ( \ell _ { j } ) } ^ { - 1 } u \left(\hat{\ell}_{i+\ell}\left(k-\sum_{t=1}^{i} r_{t} \ell_{t}+\ell_{i+1}\right)\right.\right. \\
\left.\left.=\sum_{t=i+2}^{n} \ell_{t}\right)\right\} \\
\prod_{i=1}^{n} \Delta_{\alpha_{i}\left(\ell_{i}\right)}^{-1} u\left(k+\sum_{t=1}^{n} \ell_{t}\right)-\alpha_{1}^{\left[\frac{k}{\ell_{1}}\right]+1} \prod_{i=1}^{n} \Delta_{\alpha_{i}\left(\ell_{i}\right)}^{-1} u\left(\hat{\ell}_{1}\left(k+\ell_{1}\right)+\sum_{t=2}^{n} \ell_{t}\right),(27)
\end{array}
$$

where

$$
\begin{gathered}
\sum_{(r \ell)_{1 \rightarrow i}}^{[k]}=\sum_{r_{1}=0}^{\left[\frac{k}{\ell_{1}}\right]} \sum_{r_{2}=0}^{\left[\frac{k-r_{1} \ell_{1}}{\ell_{2}}\right]} \cdots \sum_{r_{i}}^{\left[\frac{k-r_{1} \ell_{1}-r_{2} \ell_{2}-\cdots-r_{i-1} \ell_{i-1}}{\ell_{1}}\right]}, \\
\hat{\ell}_{i}(k)=k-\left[\frac{k}{\ell_{i}}\right] \ell_{i},
\end{gathered}
$$

for $i=1,2, \ldots, n, \hat{\ell}_{n+1}(k)=k, \ell_{n+1}=0$ and the operator $\prod_{j=n+1}^{n} \Delta_{\alpha_{j}\left(\ell_{j}\right)}^{-1}=1$.

Proof. From (5), we have

$$
\begin{aligned}
u(k)+\alpha_{1} u\left(k-\ell_{1}\right)+\alpha_{1}^{2} u & \left(k-2 \ell_{1}\right)+\ldots+\alpha_{1}^{\left[\frac{k}{\ell_{1}}\right]} u\left(\hat{\ell}_{1}(k)\right) \\
& =\Delta_{\alpha_{1}\left(\ell_{1}\right)}^{-1} u\left(k+\ell_{1}\right)-\alpha_{1}^{\left[\frac{k}{\ell_{1}}\right]+1} \Delta_{\alpha_{1}\left(\ell_{1}\right)}^{-1} u\left(\hat{\ell}_{1}(k)\right) .
\end{aligned}
$$

Since $\hat{\ell}_{1}\left(k+\ell_{1}\right)=\hat{\ell}_{1}(k)$, replacing $u(k)$ by $\Delta_{\alpha_{2}}^{-1} u\left(k+\ell_{2}\right), u\left(k-\ell_{1}\right)$ by

$$
\Delta_{\alpha_{2}\left(\ell_{2}\right)}^{-1} u\left(k-\ell_{1}+\ell_{2}\right) \ldots
$$

and $u\left(\hat{\ell}_{1}(k)\right)$ by $\Delta_{\alpha_{2}\left(\ell_{2}\right)}^{-1} u\left(\hat{\ell}_{1}(k)+\ell_{2}\right)$ in $(28)$, we find

$$
\Delta_{\alpha_{2}\left(\ell_{2}\right)}^{-1} u\left(k+\ell_{2}\right)+\alpha_{1} \Delta_{\alpha_{2}\left(\ell_{2}\right)}^{-1} u\left(k+\ell_{2}-\ell_{1}\right)+\ldots+\alpha_{1}^{\left[\frac{k}{\ell_{1}}\right]} \Delta_{\alpha_{2}\left(\ell_{2}\right)}^{-1} u\left(\hat{\ell_{1}}(k)+\ell_{2}\right)
$$




$$
=\Delta_{\alpha_{1}\left(\ell_{1}\right)}^{-1} \Delta_{\alpha_{2}\left(\ell_{2}\right)}^{-1} u\left(k+\ell_{2}+\ell_{1}\right)-\alpha_{1}^{\left[\frac{k}{\ell_{1}}\right]+1} \Delta_{\alpha_{1}\left(\ell_{1}\right)}^{-1} \Delta_{\alpha_{2}\left(\ell_{2}\right)}^{-1} u\left(\hat{\ell}_{1}(k)+\ell\right) .
$$

Again, replacing $\ell_{1}$ by $\ell_{2}$ and $\alpha_{1}$ by $\alpha_{2}$ in (28), we have

$$
\begin{aligned}
u(k)+\alpha_{2} u\left(k-\ell_{2}\right)+ & \alpha_{2}^{2} u\left(k-2 \ell_{2}\right)+\ldots+\alpha_{2}^{\left[\frac{k}{\ell_{2}}\right]} u\left(\hat{\ell_{2}}(k)\right) \\
& =\Delta_{\alpha_{2}\left(\ell_{2}\right)}^{-1} u\left(k+\ell_{2}\right)-\alpha_{2}^{\left[\frac{k}{\ell_{2}}\right]+1} \Delta_{\alpha_{2}\left(\ell_{2}\right)}^{-1} u\left(\hat{\ell_{2}}\left(k+\ell_{2}\right)\right) .
\end{aligned}
$$

Replacing $\mathrm{k}$ by $k-r_{1} \ell_{1}$ in (30) and multiplying both sides by $\alpha_{1}^{r_{1}}$ for $r_{1}=1,2,3, \ldots\left[\frac{k}{\ell_{1}}\right]$, we obtain

$$
\begin{aligned}
& \alpha_{1}^{r_{1}}\left\{u\left(k-r_{1} \ell_{1}\right)+\alpha_{2} u\left(k-r_{1} \ell_{1}-\ell_{2}\right) \alpha_{2}^{2} u\left(k-r_{1} \ell_{1}-2 \ell_{2}\right)+\cdots\right. \\
& \left.+\alpha_{2}^{\left[\frac{k-r_{1} \ell_{1}}{\ell_{2}}\right]} u\left(k-r_{1} \ell_{1}-\ell_{2}\right)\right\} \\
& =\alpha_{1}^{r_{1}}\left\{\Delta_{\alpha_{2}\left(\ell_{2}\right)}^{-1} u\left(k-r_{1} \ell_{1}+\ell_{2}\right)\right. \\
& -\alpha^{\left[\frac{k-r_{1} \ell_{1}}{\ell_{2}}\right]+1} \Delta_{\alpha_{2}\left(\ell_{2}\right)}^{-1} u\left(\hat{\ell}_{2}\left(k-r_{1} \ell_{1}+\ell_{2}\right)\right\} .
\end{aligned}
$$

Adding (31) for each $r_{1}=0,1,2, \ldots\left[\frac{k}{\ell_{1}}\right]$ and using (29), we arrive

$$
\begin{aligned}
& \Delta_{\alpha_{1}\left(\ell_{1}\right)}^{-1} \Delta_{\alpha_{2}\left(\ell_{2}\right)}^{-1} u\left(k+\ell_{2}+\ell_{1}\right)=\sum_{r_{1}=0}^{\left[\frac{k}{\ell_{1}}\right]} \sum_{r_{2}=0}^{\left[\frac{k-r_{1} \ell_{1}}{\ell_{2}}\right]} \alpha_{1}^{r_{1}} \alpha_{2}^{r_{2}} u\left(k-r_{2} \ell_{2}-r_{1} \ell_{1}\right) \\
& +\alpha_{1}^{\left[\frac{k}{\ell_{1}}\right]+1} \Delta_{\alpha_{2}\left(\ell_{2}\right)}^{-1} u\left(\ell_{2}+\hat{\ell}_{1}(k)\right) \\
& +\sum_{r_{1}=0}^{\left[\frac{k}{\ell_{1}}\right]} \alpha_{2}^{\left[\frac{k-r_{1} \ell_{1}}{\ell_{2}}\right]+r_{1}+1} \Delta_{\alpha_{2}\left(\ell_{2}\right)}^{-1} u\left(\hat{\ell_{2}}\left(k-r_{1} \ell_{1}+\ell_{2}\right) .\right.
\end{aligned}
$$

Continuing this process, we get (27).

The following example illustrates the existence of infinite and finite multiseries solutions of a second order mixed difference equation. 
Example 3.13. Consider the generalized mixed $\alpha_{1}$-difference equation $\Delta_{\ell_{2}} \Delta_{\alpha_{1}\left(\ell_{1}\right)} v(k)=u(k), \alpha_{1}>1, \ell_{1}, \ell_{2}>0$ and $k \in[0, \infty)$, which is the same as

$$
v\left(k+\ell_{1}+\ell_{2}\right)-v\left(k+\ell_{1}\right)-\alpha_{1} v\left(k+\ell_{2}\right)+\alpha_{1} v(k)=u(k) .
$$

From (26), the equation (33) has an infinite multi-series solution

$$
\Delta_{\ell_{2}}^{-1} \Delta_{\alpha_{1}\left(\ell_{1}\right)}^{-1} u(k)=\sum_{r_{1}=0}^{\infty} \sum_{r_{2}=0}^{r_{1}} \frac{u\left(k+\left(r_{1}-r_{2}\right) \ell_{1}+r_{2} \ell_{2}\right)}{\alpha_{1}^{r_{1}-r_{2}+1}}
$$

Now, we discuss $\Delta_{\ell_{2}}^{-1} \Delta_{\alpha_{1}\left(\ell_{1}\right)}^{-1} u(k)$ when $u(k)=k$.

Since

$$
\Delta_{\alpha_{1}\left(\ell_{1}\right)}\left\{\frac{k}{\left(1-\alpha_{1}\right)}-\frac{\ell_{1}}{\left(1-\alpha_{1}\right)^{2}}\right\}=k
$$

we get

$$
\Delta_{\alpha_{1}\left(\ell_{1}\right)}^{-1} k=\frac{k}{\left(1-\alpha_{1}\right)}-\frac{\ell_{1}}{\left(1-\alpha_{1}\right)^{2}}
$$

Similarly, since

$$
\Delta_{\alpha_{1}\left(\ell_{1}\right)} \Delta_{\ell_{2}}\left\{\frac{k_{\ell_{2}}^{(2)}}{2 \ell_{2}\left(1-\alpha_{1}\right)}-\frac{\ell_{1} k}{\ell_{2}\left(1-\alpha_{1}\right)^{2}}\right\}=k
$$

the mixed difference equation (33) for $u(k)=k$ has a closed form solution

$$
\Delta_{\ell_{2}}^{-1} \Delta_{\alpha_{1\left(\ell_{1}\right)}}^{-1} k=\frac{k_{\ell_{2}}^{(2)}}{2 \ell_{2}\left(1-\alpha_{1}\right)}-\frac{\ell_{1} k}{\ell_{2}\left(1-\alpha_{1}\right)^{2}},
$$

Taking $u(k)=k$ in $(34)$, we get

$$
\Delta_{\ell_{2}}^{-1} \Delta_{\alpha_{1\left(\ell_{1}\right)}}^{-1} k=\sum_{r_{1}=0}^{\infty} \sum_{r_{2}=0}^{r_{1}} \frac{k+\left(r_{1}-r_{2}\right) \ell_{1}+r_{2} \ell_{2}}{\alpha_{1}^{r_{1}-r_{2}+1}}
$$

which is not convergent. Hence, (33) does not have an infinite multi-series solution.

Next we consider finite multi-series solution of (33) when $u(k)=k$.

Taking $n=2, \alpha_{2}=1$ and $u(k)=k$ in Theorem 3.12, the mixed difference equation (33) for $u(k)=k$ has a finite multi-series solution of the form 


$$
\begin{aligned}
& \Delta_{\alpha_{1}\left(\ell_{1}\right)}^{-1} \Delta_{\left(\ell_{2}\right)}^{-1}\left(k+\ell_{2}+\ell_{1}\right)=\sum_{r_{1}=0}^{\left[\frac{k}{\ell_{1}}\right]} \sum_{r_{2}=0}^{\left[\frac{k-r_{1} \ell_{1}}{\ell_{2}}\right]} \alpha_{1}^{r_{1}}\left(k-r_{2} \ell_{2}-r_{1} \ell_{1}\right) \\
& \quad+\alpha_{1}^{\left[\frac{k}{\ell_{1}}\right]+1} \Delta_{\alpha_{1}\left(\ell_{1}\right)}^{-1} \Delta_{\ell_{2}}^{-1}\left(\ell_{2}+\hat{\ell}_{1}(k)\right)+\sum_{r_{1}=0}^{\left[\frac{k}{\ell_{1}}\right]} \Delta_{\alpha_{2}\left(\ell_{2}\right)}^{-1}\left(\hat{\ell}_{2}\left(k-r_{1} \ell_{1}+\ell_{2}\right),\right.
\end{aligned}
$$

where $\Delta_{\alpha_{1\left(\ell_{1}\right)}}^{-1} k$ and $\Delta_{\ell_{2}}^{-1} \Delta_{\alpha_{1\left(\ell_{1}\right)}}^{-1} k$ are given in (36) and (38). Substituting (38) in (40), we can obtain a finite multi-series solution of the mixed difference equation (33).

Similarly one can obtain closed, infinite and finite multi-series solutions of the difference equation (1) for various functions $u(k)$.

\section{Conclusion}

In general, closed form solution of the equation (1) is not possible always. When closed form solution is not possible we can go for either infinite summation form solution or finite summation form solution according to our need. If infinite series solution is not convergent, then we can find the finite series solution always. Theorem 3.11 gives an infinite multi-series solution and the Theorem (3.12) shows that the equation (1) has a finite multi-series solution for any function $u(k)$.

\section{Acknowledgments}

Research Supported by National Board for Higher Mathematics, Department of Atomic Energy, Government of India, Mumbai.

\section{References}

[1] N. R. O. Bastos, R. A. C. Ferreira, and D. F. M. Torres, Discrete-Time Fractional Variational Problems, Signal Processing, 91(3) (2011), 513-524.

[2] R. A. C. Ferreira and D. F. M. Torres, Fractional h-difference equations arising from the calculus of variations, Applicable Analysis and Discrete Mathematics, 5(1) (2011), 110-121.

[3] S. R. Grace, Oscillation of Certain Neutral Difference Equations of Mixed Type, Journal of Math Analysis Applications., 224 (1998), 241-254. 
[4] S. R. Grace and S. Donatha Oscillation of Higher Order Neutral Difference Equations of Mixed Type, Dynam Syst Appl., 12 (2003), 521-532.

[5] Jerzy Popenda and Blazej Szmanda, On the Oscillation of Solutions of Certain Difference Equations, Demonstratio Mathematica, 17(1) (1984), 153-164.

[6] Smith B and Taylor W E, Oscillation and Nonoscillation Theorems for Some Mixed Difference Equations, International Journal of Math and Sci., 15(3) (1992), 537-542.

[7] M. Maria Susai Manuel, G. Britto Antony Xavier and E. Thandapani, Theory of Generalized Difference Operator and Its Applications, Far East Journal of Mathematical Sciences, 20(2) (2006), 163-171.

[8] M. Maria Susai Manuel, V. Chandrasekar and G. Britto Antony Xavier, Solutions and Applications of Certain Class of $\alpha$-Difference Equations, International Journal of Applied Mathematics, 24(6) (2011), 943-954.

[9] K. S. Miller and B. Ross, Fractional Difference Calculus in Univalent Functions, Horwood, Chichester, UK, (1989), 139-152.

[10] M. Maria Susai Manuel, G. B. A Xavier, V. Chandrasekar and R. Pugalarasu, Theory and application of the Generalized Difference Operator of the $\mathrm{n}^{\text {th }}$ kind(Part I), Demonstratio Mathematica, 45(1) (2012), 95-106.

[11] M. Maria Susai Manuel, V. Chandrasekar and G. Britto Antony Xavier, Theory of Generalized $\alpha$-Difference Operator and its Applications in Number Theory, Advances in Differential Equations and Control Processes, 9(2) (2012), 141-155.

[12] E. Thandapani and N. Kavitha, Oscillatory Behaviour of Solutions of Certain Third Order Mixed Neutral Difference Equations, Acta Mathematica Scientia, 33B(1) (2013), 218-226. 
